Check for updates

Cite this: Mater. Adv., 2022, 3, 3199

Received 19th September 2021 Accepted 7th December 2021

DOI: $10.1039 / \mathrm{d} 1 \mathrm{ma} 00863 \mathrm{c}$

rsc.li/materials-advances

\section{Polylactic acid 3D interconnected nanonetworks as high reflectance distributed Bragg reflectors}

\begin{abstract}
Pedro M. Resende (D) and Marisol Martín-González (D) *
Natural systems found ways to exploit light at the nanoscale, devising complex 3D structures that behave as photonic crystals, able to produce structural coloration. Distributed Bragg reflectors are a particular example of 1D photonic crystals, used in different applications, including structural coloration. Currently distributed Bragg reflectors rely on multi-material deposition or material doping to achieve high refractive index contrast, unlike the biological counterparts that often rely on single-materials through structural variations. In this work, we report single-material polymeric distributed Bragg reflectors fabricated from the infiltration of polylactic acid into 3D anodic aluminium oxide templates. These templates act as sacrificial scaffolds for the nanostructuration of the polymeric material, generating a 3D polymer network with periodic modulations. The obtained structures present very high reflectance, above $95 \%$, with a low number of periods around 20 repeating layers. These structures offer a new approach to the generation of flexible single-material DBRs with high reflectance, an important issue in all-polymer photonic systems.
\end{abstract}

\section{Introduction}

Many biological systems in nature are imbued with hierarchical structures that confer environmental advantages and ensure survivability. Several species of insects, such as butterflies and beetles, and several plants, present repeating hierarchical structuration able to produce strong structural coloration. The exploitation of light by these species has long intrigued scientists leading to the development of a wide array of photonic structures for coloration, light guiding, and light manipulation. ${ }^{1-3}$ Distributed Bragg reflectors (DBRs) are an example of this type of photonic structure, corresponding to 1D photonic crystals. DBRs present a layered structure with periodic modulation of the refractive index, which is responsible for the observed photonic stop band. The photonic response of these structures can be tailored by controlling the materials and layer periodicity, presenting strong responses across a vast range of the electromagnetic spectrum, including the visible range. These properties led to the application of DBRs in, e.g., sensing, photovoltaics, solar concentrators, and highly reflective mirrors. ${ }^{4-9}$ Typically, DBRs have been produced through physical deposition of high refractive index inorganic materials, such as $\mathrm{TiO}_{2}, \mathrm{SiO}_{2}, \mathrm{SnO}_{2}, \mathrm{ZnO}$, etc. ${ }^{10,11}$ These methods generally require costly infrastructure and have low compatibility with large-area fabrication or the use of flexible substrates. Employing nanoparticle dispersions of high permittivity oxides directly addressed the higher cost besides

Instituto de Micro y Nanotecnologia, IMN-CNM, CSIC (CEI UAM + CSIC), Isaac Newton, 8, E-28760, Tres Cantos, Madrid, Spain. E-mail: marisol.martin@csic.es being compatible with several solution coating techniques. Nevertheless, flexibility, stretchability, or bendability are also desired properties for the new generation of flexible organic devices. Different approaches have been used to improve mechanical properties, which include: (i) embedding nanoparticle dispersions into polymer matrixes or an intermediate polymer/ inorganic composite, allowing the modulation of the dielectric properties of the individual layers; ${ }^{6,12-18}$ (ii) deposition of thin oxides into flexible substrates; ${ }^{5,7-9}$ (iii) and the use of polymeric dielectric media, through the use of amorphous homopolymers, block copolymers, or liquid crystals. ${ }^{1,2,4,19-25}$

These approaches have allowed the coupling of inorganic DBRs into flexible systems, the generation of hybrid composites that neatly integrate into organic devices or the complete replacement of inorganic constituents with flexible polymeric materials. However, hybrid systems and composites raise other concerns, particularly long-term operation under stress, or conformational coating. On the one hand, when inorganic films are coupled with flexible substrates, continued stress may lead to DBR deterioration, decreasing the device's overall performance. On the other hand, hybrid composites may suffer from deterioration due to fracture formation or polymer matrix degradation. For hybrid materials, the post-use processing should also be considered, as the combination of different materials makes it difficult to perform separation, disposal, or recyclability. Despite this, these systems were already integrated into flexible systems, such as photovoltaics, OLEDs, or solar concentrators; $;^{5,7-9,15}$ and in sensing applications relying on the selective swelling of polymeric layers. ${ }^{6,16}$ Finally, allpolymer systems managed to tackle many of the issues found in 
inorganic films or hybrid materials, but still present low refractive index contrast, requiring a high number of layers in the DBR to attain strong photonic responses. All-polymer DBRs opened new possibilities for the functionalization of these structures for sensing. Examples include the use of hydrogels, block copolymers, and liquid crystals with selective response to different chemical and physical stimuli, such as vapors, acids, or electric fields, resulting in stopband shifts from selective layer swelling. ${ }^{2,6,17,26,27}$

Some works have proposed single-polymer or singlematerial DBRs reducing material usage and bypassing the need for later material separation. ${ }^{6,15,28}$ Unfortunately, these works still rely on the same doping agents, such as oxide nanoparticles, to obtain higher refractive index contrasts. True single-material polymeric DBRs would result in flexible and bendable structures with a simple after-use disposal procedure that could be coupled with many of the current organic device systems. Polymer nanostructuration into porous networks constitutes an approach to single-material DBRs by generating porosity modulations that result in refractive index variations. This can be done through hard-template nanostructuration, such as $3 \mathrm{D}$ anodic aluminium oxide (AAO) templates. AAO templates have been extensively used in polymer nanostructuration and research, due to their ease of fabrication and application, and their compatibility with many different polymer infiltrations and synthesis procedures. ${ }^{29,30}$ Remarkably, these templates were also investigated as stand-alone DBRs, either through the deposition of metals or in-depth modulation of refractive index. ${ }^{31-33}$ Their continued research led to the development of 3D AAO templates, as photonic structures and scaffolds for material nanostructuration. In particular, these structures opened new research opportunities for the generation of 3D polymeric architectures. ${ }^{34}$ Only a few works have explored the use of 3D AAO for polymer nanostructuration, which motivates the application of these structures and the combination of novel polymeric materials. ${ }^{3,34-37}$ Our group has previously reported on the fabrication of these structures from polyethylene, a commodity thermoplastic, resorting to polymer melt infiltration. ${ }^{3}$ The final structures presented 3D architectures that resulted in structural coloration. The same approach was later used to obtain insight into polymer organization inside these new 3D confinement geometries. ${ }^{35}$ Despite the bright colorations that were obtained in these works, the high degree of crystallinity of polyethylene lead to strong light scattering, deterring these structures from being applied as high-performance photonic components, but rather finding the best use in coloration applications. In this sense, there is still a need for the employment of different polymeric materials with different properties, such as low crystallinity or amorphous, intrinsic physical properties, or the use of more environmentally-friendly polymers. Demonstrating the feasibility of these 3D AAO templates for polymer nanostructuration can help propel their application in the fabrication of high-performance photonic systems entirely composed of polymeric materials.

Polylactic acid (PLA) presents itself as a good proof-ofconcept candidate due to its biodegradable and biocompatible properties. PLA is a thermoplastic polymer produced from the ring-opening polymerization of lactide. ${ }^{38,39}$ This material offers other advantages compared to other commonly used homopolymers: production from renewable sources; consumption of carbon dioxide during fabrication; processability and recyclability. ${ }^{39}$ PLA was already the subject of several fundamental polymer studies, ${ }^{40-47}$ the development of composites, and the application in different areas including, e.g., drug delivery or cell culture, ${ }^{48,49}$ sensing and energy harvesting, ${ }^{50-55}$ and flexible electronics and microfluidics. ${ }^{56-58}$ Within photonic applications the majority of published works focused on the fabrication of waveguides and gratings. ${ }^{59-63}$ The resulting photonic structures in these works demonstrated good reflectivity around near to far IR, but with no examples within the visible spectrum. ${ }^{61,64}$ The fabrication of PLA DBRs would result in true single-material photonic structures that could directly be applied in several flexible and organic devices, such as solar cells, photovoltaics, solar concentrators, OLEDs, or their use as highly reflective coatings and generation of structural coloration. ${ }^{65}$ The additional biocompatibility and biodegradability of PLA also bring the possibility to combine these structures with biological functions, such as in situ monitoring, cell culture, tissue engineering, and light delivery and sensing. ${ }^{58,60,62}$

In this work, we report the fabrication of single-polymer and single-material DBRs based on 3D PLA nanonetworks, by the implementation of polymer nanostructuration with 3D AAO templates. The development of these nanonetworks resorts to an accessible and easy template fabrication technique, based on a current density pulsed anodization approach, followed by the melt infiltration of PLA. The resulting PLA DBRs (3D polymeric nanonetworks - NNs) present strong structural coloration and high-reflectance along the visible spectrum, demonstrating the possibility to fabricate PLA-based photonic structures from the simple anti-replication of sacrificial 3D inorganic templates, without resorting to the use of doping agents or coupling with high refractive index inorganic materials in the final structure.

\section{Experimental section}

\section{Reagents and materials}

High purity aluminium sheets (Al, 99.999\% wt) for anodization were purchased from Advent Research Materials (UK). Polylactic acid (PLA, granules with $0.3 \mathrm{~mm}$ average diameter) for the melt infiltration was purchased from Goodfellow Ltd. (UK). Acetone and isopropanol were purchased from Carlo Erba Reagents (Spain). Ethanol absolute was purchased from PanReac AppliChem. Sulfuric acid $\left(\mathrm{H}_{2} \mathrm{SO}_{4}, 96 \%\right)$ was purchased from J. T. Baker. Perchloric Acid $\left(\mathrm{HClO}_{4}, 70 \%\right)$ was purchased from Alfa Aesar. Copper Chloride $\left(\mathrm{CuCl}_{2}, 97 \%\right)$ was purchased from Aldrich. Orthophosphoric Acid $\left(\mathrm{H}_{3} \mathrm{PO}_{4}, 85 \%\right)$ was purchased from Prolabo.

\section{D AAO templates}

The used 3D AAO templates were produced following a galvanic anodization process in a $1.1 \mathrm{M} \mathrm{H}_{2} \mathrm{SO}_{4}(25 \% \mathrm{v} / \mathrm{v}$ ethanol) solution. In this method, a periodic square-wave current 
density profile is applied during anodization, alternating between a high and low value of current density. This method produces intercalated layers of nanopores with different diameters and porosities. The gradual etching of the smaller diameter nanopores (grown under low current density) leads to the connection of adjacent pores, and ultimately to the formation of a transversal interconnection, perpendicular to the vertical nanopores. This process results in 3D AAO templates with strong structural coloration. A detailed description of this method and the used parameters can be found in the literature. ${ }^{3,35}$ The aluminium substrates were cleaned in ultrasonic baths of acetone, deionized water, isopropanol, and ethanol, each for the duration of 5 minutes, before performing an electropolishing process. Electropolishing was performed in an $\mathrm{HClO}_{4}$ and Ethanol solution (1:3 ratio in $\mathrm{v} / \mathrm{v}, \mathrm{HClO}_{4}$ : Ethanol), under $10{ }^{\circ} \mathrm{C}$ and vigorous stirring, by applying a constant potential of $20 \mathrm{~V}$ for 5 minutes. The substrates were then immersed in ethanol and deionized water and finally dried with an air-gun. The galvanic anodizations and the chemical etching were performed for 3 days: in the first 1.5 days, the substrates were actively anodized after which they were kept in contact with the electrolyte for the remaining 1.5 days at zero current density. This approach ensures the formation of the transversal connections typical in these 3D AAO templates while maintaining good template uniformity. The anodization electrolyte was kept at $-1{ }^{\circ} \mathrm{C}$. After the anodization process, the samples were removed from the electrolyte, cleaned with deionized water, and dried with an air-gun. In this work, three different profile periodicities were used that resulted in 3D AAO templates with different photonic responses within the visible part of the spectrum. The produced templates presented reflectance maxima at 465,535 , and $597 \mathrm{~nm}$, visually corresponding to blue, green, and orange respectively.

\section{PLA infiltration}

PLA was infiltrated inside the 3D AAO templates through melt infiltration. Polymer melt infiltration has been extensively used in the literature for the fabrication of polymeric nanowires and nanotubes. ${ }^{29,66}$ The polymer is heated above its fusion temperature and allowed to spontaneously flow inside the cavities/ pores of the template. PLA granules were first melted into a film with an average thickness of $0.5 \mathrm{~mm}$ by placing the granules on a glass slide and heating the polymer for a few seconds at $170{ }^{\circ} \mathrm{C}$ in a hot plate while applying constant pressure. The polymer film was then quenched by placing the glass slides on top of a metallic heat dissipater, kept under $0{ }^{\circ} \mathrm{C}$. The resulting films were then cleaned in an ultrasonic bath of ethanol for a duration of 6 minutes. The melt infiltration process was then performed by placing the PLA films on top of the AAO templates and storing them inside a low-vacuum desiccator chamber equipped with a heating plate. The infiltration was performed at $170{ }^{\circ} \mathrm{C}$ and low pressure ( $c a .100$ mbar) for the duration of 4 hours. During infiltration, constant contact pressure was applied with a metallic cylinder to flatten and spread the molten polymer across the entire AAO surface. The samples were then removed from the desiccator and left to cool down to room temperature. The amount of PLA used for each infiltration was in excess (the thickness of the PLA films was much greater than that of the 3D AAO templates), ensuring there was enough bulk material to fill the templates. The remaining bulk material after the infiltration was not removed, serving as support for the PLA nanonetworks.

\section{PLA nanonetworks}

To obtain the polymeric nanonetworks two additional etching steps were performed after the infiltration process, to remove the $\mathrm{Al}$ substrate and the AAO template. The aluminium substrate was first removed in an aqueous solution of $\mathrm{CuCl}_{2}$ and $\mathrm{HCl}$ $(10.8 \mathrm{~g}$ and $150 \mathrm{~mL}$, respectively, total solution volume of $450 \mathrm{~mL}$ ), followed by 3D AAO template dissolution using an aqueous solution of $\mathrm{H}_{3} \mathrm{PO}_{4}(10 \% \mathrm{wt})$ at room temperature, for a minimum of $12 \mathrm{~h}$. During template dissolution, structural coloration could be gradually observed. After the complete removal of the template, the samples were removed, cleaned with deionized water, and dried with an air-gun, after which they presented strong coloration.

\section{Characterization techniques}

Scanning Electron Microscopy (SEM) imaging was performed with an FEI Verios 460 HR-SEM. The observation was done under low current and voltage settings (13 pA and $1.3 \mathrm{kV}$, respectively), fast acquisition, and scanning interlace, avoiding structural damage due to polymer melting. For cross-section imaging, the different samples were immersed in liquid nitrogen and then fractured by quickly cutting the samples with a sharp and clean blade. Spectrophotometry was performed with a UV-vis spectrometer from PerkinElmer (Lambda 950 UV-vis), equipped with an Integrated Sphere module. This module allows the measurement of the total and diffuse reflectance spectra in direct light incidence $\left(\right.$ at $8^{\circ}$ ). The total spectra are measured from the total reflected indecent beam that exits the main chamber aperture. The diffuse component is measured through a small side-aperture, roughly at $45^{\circ}$ relative to the sample normal. For a perfectly flat and specular surface, the diffuse component is zero. The specular component was extracted by subtracting the diffuse spectrum from the total reflectance spectrum. The measurements were performed relative to a high reflectance spectrophotometry standard (Spectralon, corresponding to $100 \%$ reflectance), with a beam spot size of $10 \mathrm{~mm}$ per $5 \mathrm{~mm}$. The measured spectra were within the 200 to $1000 \mathrm{~nm}$ range, after which the maxima of reflection of each sample was extracted.

\section{Results and discussion}

The preparation of all-PLA DBRs (NNs) started with the fabrication of several 3D AAO templates with different reflectance responses in the visible spectrum, in this case, around blue, green, and orange, with average maxima, $\lambda_{3 \mathrm{D} \text { AAO }}$, at 465,535 , and $597 \mathrm{~nm}$ respectively. These templates were subsequently used in the melt infiltration of PLA, by placing previously prepared PLA films on top of the templates, and keeping the polymer and template at 
$170{ }^{\circ} \mathrm{C}$ for 4 hours and under low vacuum, as described in the Methods section. During the melt infiltration process, the molten PLA flows inside the 3D AAO template filling the nanopores and cavities. The presence of periodically spaced transversal interconnections (perpendicular to the vertical pores typically found in AAO templates) in these templates allow the polymer to flow into the sides, forming a porous horizontal PLA layer that connects adjacent polymeric material. Through this method, a fully 3D polymer network can be obtained. An excess of PLA was used to ensure the templates were fully infiltrated, in addition to providing support for the PLA NN through the remaining bulk film. After the infiltration process, the samples were left to cool down naturally to room temperature. To finally obtain the PLA NN, several etching procedures were applied, starting with the chemical removal of the remaining Al substrate from the templates, followed by chemical dissolution of the oxide with a dilute phosphoric acid solution, effectively releasing the polymeric NNs from the templates. A strong coloration could be immediately observed after the complete dissolution of the template. The chemical processing step did not introduce any visible degradation to the polymer. This is illustrated in Fig. 1a, starting with the polymer infiltration and followed by chemical etching and release of the PLA NN. The generated NNs are shown in Fig. 1b, below the originally employed 3D AAO templates. The PLA NNs presented strong structural coloration, visibly corresponding to blue, green, and orange wavelengths, with measured maxima, $\lambda_{\text {PLA NN }}$, at 498, 546, and $600 \mathrm{~nm}$, respectively.
A wavelength shift from the used 3D AAO to the resulting PLA NN was observed, being more significant for lower wavelengths. A difference in coloration intensity can also be observed between the original 3D AAO templates and the obtained PLA NN, suggesting the resulting PLA DBRs present a stronger coloration. Physically, aluminum oxide (alumina) has a higher refractive index than PLA, and it should be expected that the higher refractive index contrast of alumina/air would produce more intense reflections. The apparent discrepancy in the observed intensity can be explained by the presence of background material, in this case, the aluminum substrate below the $3 \mathrm{D}$ AAO templates that acts as a highly reflective mirror. The perceived intensity depends not only on the value of the reflectance maxima but also on the background reflectance (analogous to signal-tonoise ratio). The high reflectivity of the aluminum surface across the entire visible range contributed to the analyzed spectrum, decreasing the perceived intensity. In the case of the PLA NN, as there was no reflective background material (in this case a black non-reflective surface), the transmitted light was absorbed or scattered in other directions and was not reflected. From Fig. 1b, a remaining ring of $\mathrm{Al}$ can also be seen in these images, which served as additional support for the PLA film and NNs, allowing easy sample manipulation. Despite this, the PLA film/ PLA NN could be separated from the supporting $\mathrm{Al}$ and be easily manipulated without damage, as shown in Fig. 1c. In this image, it can be seen that the intermediate stage composed by the PLAfilled 3D AAO is transparent, without any colored reflections.
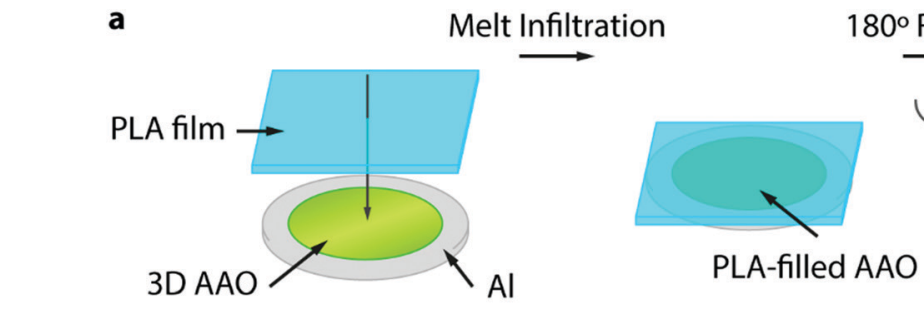

\section{$80^{\circ}$ Rotation Chemical Etch}
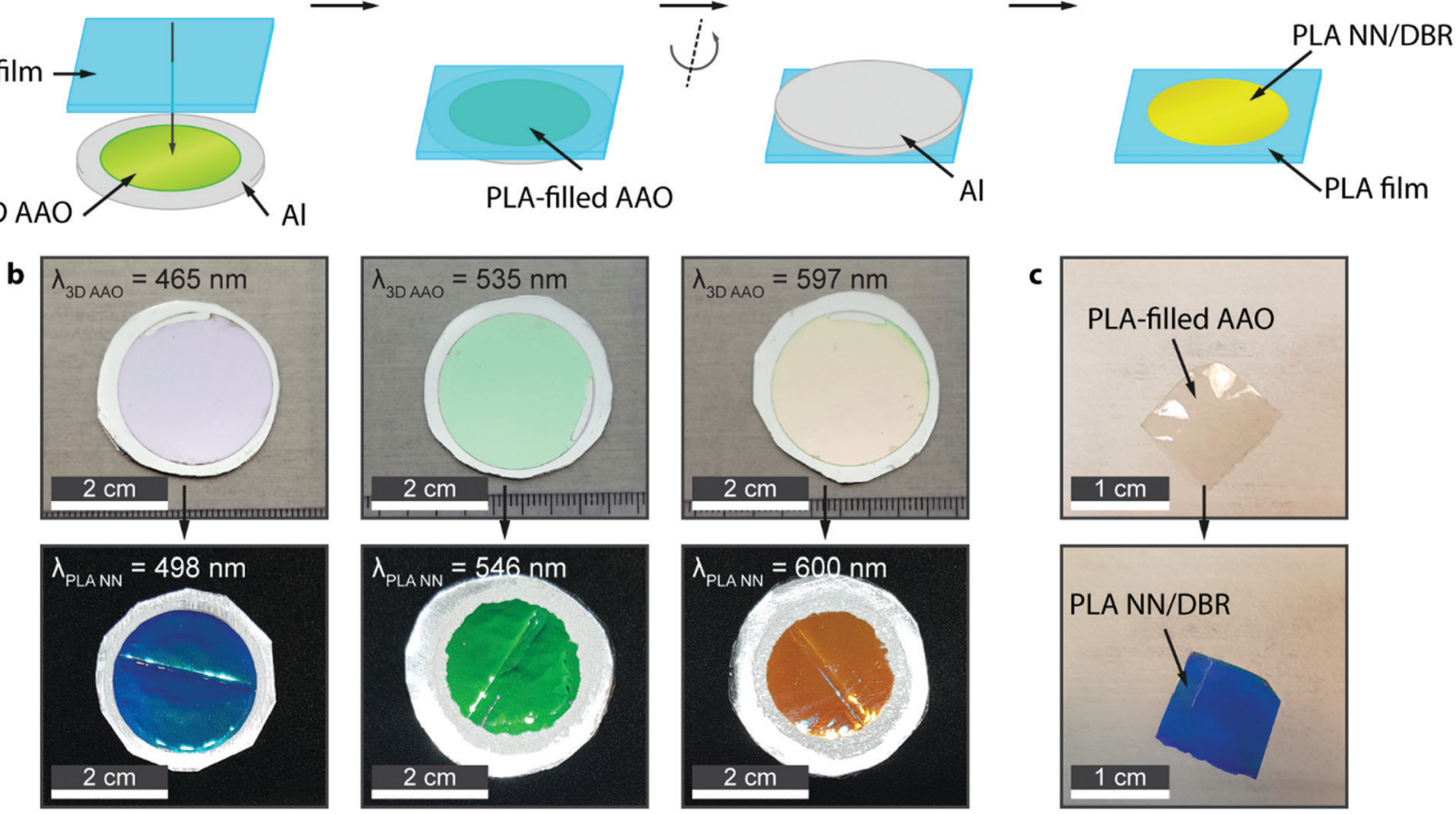

Fig. 1 The fabrication method and resulting PLA NN. (a) Fabrication method of the PLA NN, starting with polymer melt infiltration, followed by removal of the Al substrate and finally chemical etching of the AAO template, releasing the NN/DBR. (b) Images of the used 3D AAO templates, presenting structural coloration in the blue, green, and orange parts of the visible spectrum, and the corresponding obtained PLA NN, also reflecting in the blue, green, and orange parts of the visible spectrum. (c) Sample of PLA-filled AAO separate from the supporting substrate, before and after the AAO removal, presenting coloration only after removing the template. 
The coloration can only be observed after the chemical etching of the template, releasing the PLA NN, as shown in the bottom image of Fig. 1c.

The reflectance spectra of the produced PLA NNs were assessed with spectrophotometry measurements. Fig. 2 shows the different obtained spectra and the respective relationship between the used templates and the resulting NNs. In particular, Fig. 2a, shows the total, specular, and diffuse reflectance spectra for the orange PLA NN. In this case, the first-order reflection can be observed at $c a .600 \mathrm{~nm}$, with a second-order peak also visible at $302 \mathrm{~nm}$, corresponding to half the wavelength of the firstorder peak. The total reflectance at the first-order peak was observed to reach almost $100 \%$, in accordance with the visibly high reflectivity of these samples. The measured diffuse component presented overall very low values (around $2 \%$ ), with a peak below $20 \%$ that followed the same trend as the total obtained spectra. The similar shape of the diffuse spectrum (Fig. 2a), including the increase around the peak maxima, can be explained by the surface roughness and thickness variations of the bulk PLA support, as seen in Fig. 1b. These surface defects present on the bulk support led to an increase in light scattering, increasing the diffuse reflections at the interfaces. A decrease of the diffuse component could be achieved by decreasing surface roughness through a finer control on the surface melt spread ensuring a more consistent thickness for the bulk support film, and so, decreasing light scattering at the interfaces. Despite this, the diffuse component is observed to be very low across the entire visible spectrum likely due to the low crystallinity of PLA, a behavior that is expected considering the used templates present very small pore sizes (below $20 \mathrm{~nm}$, on average) that hinder polymer crystallinity, as well as the absence of any thermally induced crystallization process post-infiltration. The total reflectance spectra for the obtained samples are plotted in Fig. 2b, where the gradual red-shift of the first-order reflection peak can be observed from the blue to the orange samples $(498,546$, and $600 \mathrm{~nm}$ ). This shift agrees with the initial 3D AAO templates, with 3D AAO having longer wavelengths resulting in PLA NNs with responses also at longer wavelengths, pointing to a good correspondence between the obtained coloration, with low deviation, and the anti-replication of the 3D network structure. In addition to the first-order peaks, second-order reflections can also be seen on the left side of the plots, presenting the same behavior. The observed behavior and the presence of secondorder peaks can be predicted from the Bragg-Snell equation: ${ }^{67,68}$

$$
m \lambda=2 d \sqrt{n_{\mathrm{eff}}^{2}-n_{\mathrm{air}}^{2} \sin ^{2} \theta}
$$

where $n_{\text {eff }}$ is the layer effective refractive index, $n_{\text {air }}$ is the refractive index of air $(\approx 1), d$ is the thickness of the layer, $\theta$ is the angle of incidence with respect to the normal of the surface, $m$ is an integer (reflection order), and $\lambda$ is the wavelength of the incident/reflected light. From the equation, it can be seen the reflected wavelength $\lambda$ has a direct dependence on the layer period/thickness $d$. The equation can also predict the location of high-order reflections. Under the same conditions, i.e., same refractive index, layer thickness, and angle of incidence, the equation can only be satisfied if the reflected wavelength equals $\lambda / m$. This means that a second-order reflection, corresponding to $m=2$, will be observed at a wavelength of $\lambda / 2$, at half the first-order peak. From this argument, it can be concluded that the observed main peaks in Fig. $2 \mathrm{~b}$, with high reflectance, correspond to first-order reflections, while the peaks observed at the left-most side of the spectra correspond to second-order reflections, with maxima around $\lambda / 2$. All the measured samples presented first-order peak reflectance values above 95\%. In Fig. 2c, a plot is shown relating the 3D AAO template reflection maxima $\left(\lambda_{3 \mathrm{DAAO}}\right)$, with the maxima obtained for the resulting PLA NNs ( $\left.\lambda_{\text {PLA NN }}\right)$. The graph shows a linear relationship between these variables, with a good linear fit (equation shown in the graph), allowing the determination of the PLA NN coloration based on the maxima of the 3D AAO templates.

Lastly, the morphology of the generated PLA NNs was assessed through SEM imaging. Several SEM micrographs can be seen in Fig. 3, showing the cross-section and top-view of the obtained PLA NN orange sample. In Fig. 3b a cross-section is shown for the intermediate stage corresponding to the PLAfilled 3D AAO, with an average thickness of $5.1 \mu \mathrm{m}$. From the micrograph, an estimation of the number of repeating layers
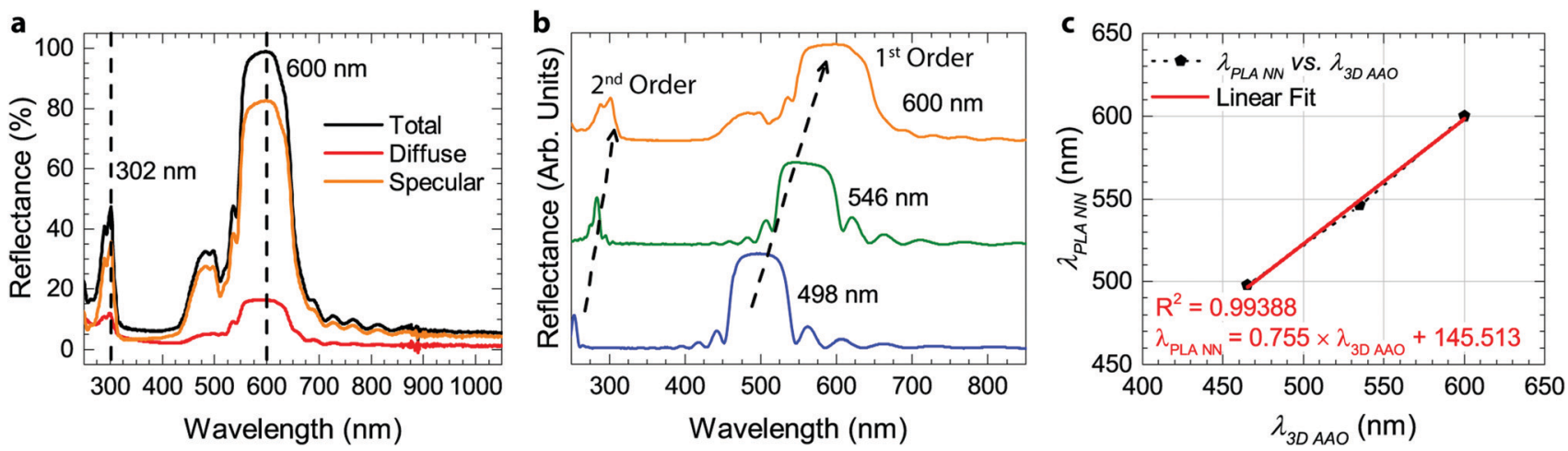

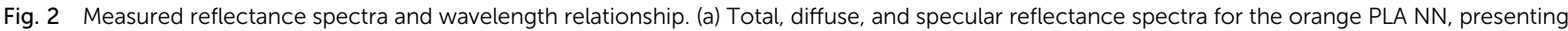

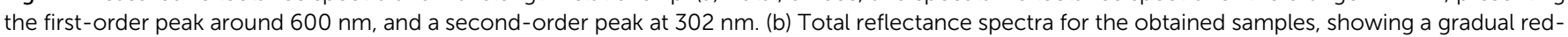

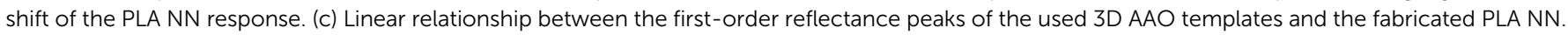



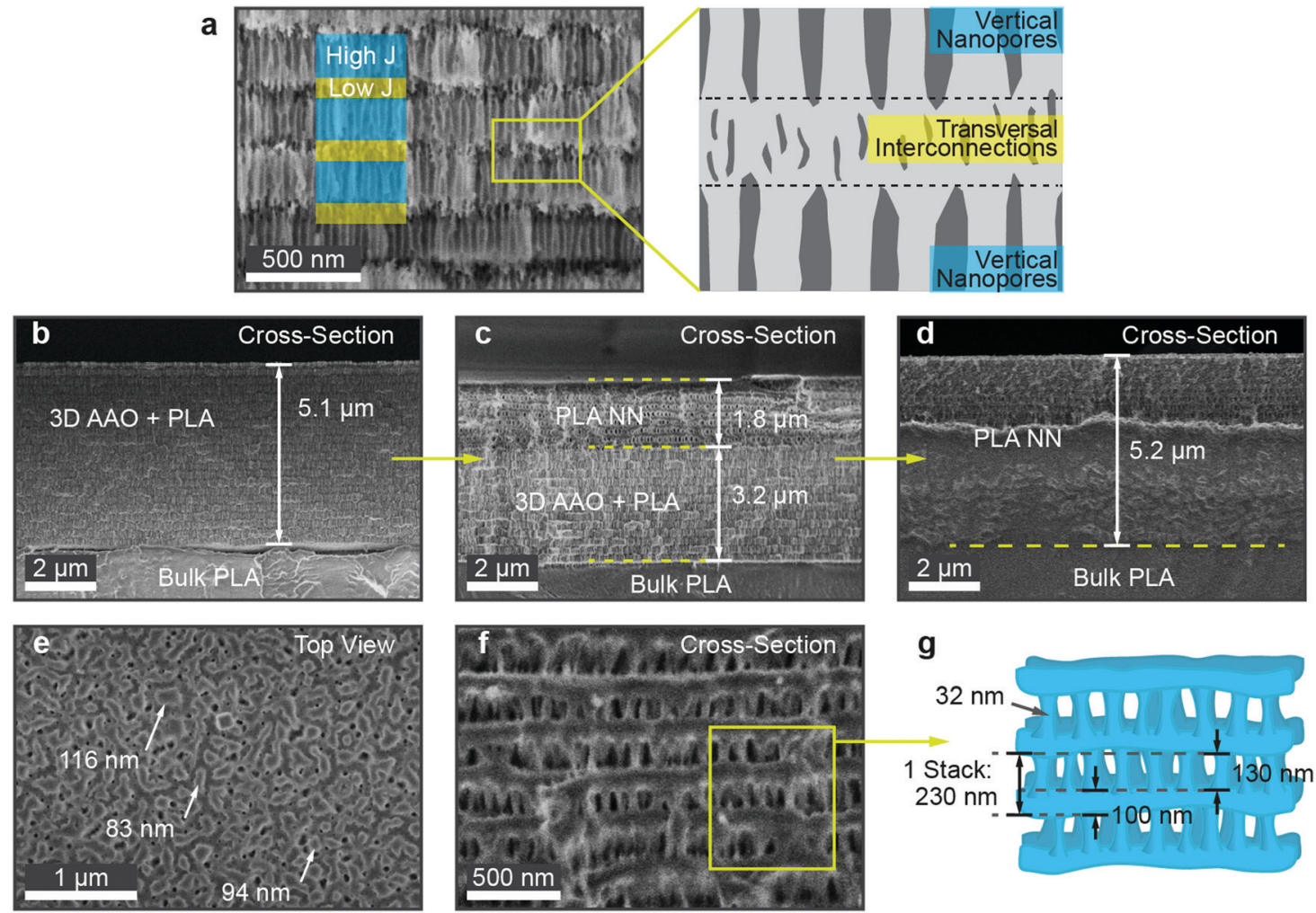

Fig. 3 SEM characterization and structure scheme. (a) Microstructure of a 3D AAO template and respective scheme: the structure is composed of two distinct layers of vertical nanopores (corresponding to the blue areas, grown under high current density - High J) and transversal interconnections (yellow areas, grown under low current density - Low J), effectively forming a bilayer stack. (b) Cross-section SEM micrograph of a PLA-filled 3D AAO (orange PLA NN), with an average thickness of $5.1 \mu \mathrm{m}$. (c) Cross-section of the sample in (b), after a 7 hour etching process, showing the partial dissolution of the template and release of the PLA NN. (d) Cross-section of the PLA NN after complete template removal, with average thickness around $5.2 \mu$ m. (e) Top-view micrograph of the same sample, presenting pillar-like formations with sizes ranging from 94 up to $116 \mathrm{~nm}$. (f) High magnification crosssection of the sample shown in (d), presenting a network-like morphology. (g) Scheme illustrating the 3D structure of the PLA NNs observed in ( $f$ ), composed by a bilayer stack $(230 \mathrm{~nm})$ : a layer of vertical nanowires $(130 \mathrm{~nm})$ connected to horizontal porous layers of polymer (100 $\mathrm{nm})$.

(or periods) can be made, yielding around 22 periods. Fig. 3c shows the cross-section of the same sample after being immersed in a phosphoric acid-based etchant for 7 hour, slowly releasing the PLA NN from the oxide. The completely released PLA NN can be seen in Fig. 3d, in which part of the crosssection can also be seen to be covered by the bulk polymer. This is due to the plastic deformation of the polymer during sample preparation for SEM imaging. Nevertheless, from these images an estimation of the total thickness can still be obtained, resulting in an average thickness of $5.2 \mu \mathrm{m}$. This value is approximately the same observed in the micrograph in Fig. $3 \mathrm{~b}$ suggesting that the etching procedure and the gradual release of the NN did not induce polymer expansion or vertical swelling, which would increase the final total thickness of the nanostructures. A higher magnification image of the PLA NN structure is shown in Fig. 3f, showing in detail the observed layeredstructure. A scheme is shown in Fig. 3g, illustrating the observed morphology of these NN (in Fig. 3f) composed by a bilayer stack $(230 \mathrm{~nm})$ : a layer of vertical nanowires $(130 \mathrm{~nm})$ connected to a horizontal porous layer of polymer $(100 \mathrm{~nm})$. This structure corresponds to the anti-replication of the vertical nanopores and horizontal interconnections respectively, as seen in Fig. 3a. The measurements shown in Fig. $3 \mathrm{~g}$ of the stack period (and individual layer thickness) correspond to the values obtained for the PLA NN orange sample (shown in Fig. 3b-f). The average thickness of the vertical nanowires was measured to be around $32 \mathrm{~nm}$, which is slightly above the expected nanopore sizes for the fabricated 3D AAO templates, typically ranging from 10 up to $20 \mathrm{~nm}^{3,35,69}$ This suggests that polymer relaxation takes place at the nanowire level, slightly increasing their diameter without changing the total thickness of the NNs. A top-view micrograph of the PLA NNs can also be seen in Fig. 3e presenting vertical pillar-like formations, with sizes ranging from 94 up to $116 \mathrm{~nm}$. Given that the average nanowire diameter is around $32 \mathrm{~nm}$, it can be concluded that the pillar-like formations observed in these top-view micrographs correspond to bundles of nanowires, that likely collapsed due to surface tension.

The performed SEM characterization successfully shows that the observed coloration of these structures is a consequence of the periodic nanostructuration, as observed in Fig. 3. Furthermore, the obtained reflectance spectra can reach values above $95 \%$ despite the low number of periods, which for the observed case was only 22 periods. This is an important result considering the typical low refractive index contrast that polymeric systems present. DBR stacked-layer morphologies composed of polymeric materials tend to require several tens of stacks to obtain strong structural coloration and in many 
cases the use of composite layers through the addition of high refractive index inorganic materials. Lastly, the approximate NN porosity was also estimated, as this constitutes an important parameter for applications such as sensing, filtration, biological applications like cell cultures and tissue engineering, and the fabrication of composite materials. The porosity was calculated based on the sample mass variation before and after the chemical etching of the AAO template. As the fabricated 3D AAO templates do not present well-ordered nanopore arrays and are composed of a random spatial distribution of pores, it becomes difficult to determine a value of porosity from simple geometrical arguments. ${ }^{35,69}$ This difficulty justified the approximation of the porosity through an indirect method, i.e., the use of the mass variation before and after AAO chemical etching. For the case of the PLA NNs, the porosity introduced from nanostructuration corresponds to the volume fraction of AAO. This volume is later replaced by air after the AAO chemical dissolution. The measurement of mass variation allowed the determination of the AAO mass that was present for the considered sample. The volume occupied by the 3D AAO could then be estimated through the density value of amorphous $\mathrm{Al}_{2} \mathrm{O}_{3}$, which for sulfuric-acid based AAOs was reported to be in the order of $2.8 \mathrm{~g} \mathrm{~cm}^{-3} \cdot{ }^{70}$ The sample AAO mass (mass variation) for the sample shown in Fig. 1c was determined to be $0.61 \mathrm{mg}$, which corresponded to an AAO volume of $c a .2 .179 \times 10^{-4} \mathrm{~cm}^{3}$. The total volume occupied by the PLA NN and the AAO was also calculated, based on the surface area of the sample $\left(1.064 \mathrm{~cm}^{2}\right)$ and the thickness (from Fig. 3a, $5.1 \mu \mathrm{m}$ ), resulting in a value of $5.43 \times 10^{-4} \mathrm{~cm}^{3}$. The AAO volume fraction can then be determined as the ratio of the AAO volume by the total volume, $\left(2.179 \times 10^{-4} / 5.43 \times 10^{-4}\right) \times 100=40.15 \%$. As mentioned, after the chemical removal of the $\mathrm{AAO}$, the volume that was previously occupied by the amorphous $\mathrm{Al}_{2} \mathrm{O}_{3}$ is replaced by air, and assuming negligible polymer expansion, it can be concluded that the porosity of the fabricated PLA NNs is around $40 \%$.

\section{Conclusions}

In this work, we have demonstrated the fabrication of singlepolymer/single-material PLA-based distributed Bragg reflectors, through the anti-replication of sacrificial 3D inorganic templates (3D AAO). A linear relationship was observed between the coloration of the used templates and the resulting PLA DBRs, allowing the determination and tailoring of the DBR photonic response. Very high total reflectance values were obtained for the fabricated samples, with values above 95\% despite the low number of layers (around 20 periods/stacks), which represents an important result, as polymeric materials typically present very low refractive indexes and low contrast in photonic applications. This method successfully circumvents this issue, without resorting to high refractive index additives or the fabrication of a hybrid or composite material. The obtained PLA DBRs also presented very low diffuse reflectance across the visible range, likely due to the low crystallinity of PLA under such small nanoconfinement geometries. This represents an important step for the application of these structures as high-performance photonic components, presenting low light scattering. The observed morphologies through SEM imaging confirmed that the observed coloration arises from the nanostucturation of the polymeric material. Lastly, an estimation of the NN porosity was performed through the sample mass variation from AAO dissolution, yielding a value of around $40 \%$. These results open the possibility to employ these structures in biological systems, like membranes, drug delivery, monitoring and sensing, with the additional benefit of presenting biodegradable properties.

\section{Author contributions}

P. M. R. performed the experiments and sample preparation. P. M. R. also performed optical imaging, SEM imaging, spectrophotometry measurements, and subsequent analysis. M. M. G. encouraged and supervised the work. All authors contributed to the manuscript.

\section{Conflicts of interest}

There are no conflicts to declare.

\section{Acknowledgements}

M. S. M. G wants to acknowledge the MINECO project PID2020118430GB-100 for financial support. The authors acknowledge also the service from the MiNa Laboratory at IMN, and funding CM (project SpaceTec, S2013/ICE2822), MINECO (Project CSIC13-4E-1794), and EU (FEDER, FSE).

\section{Notes and references}

1 M. Kolle, A. Lethbridge, M. Kreysing, J. J. Baumberg, J. Aizenberg and P. Vukusic, Adv. Mater., 2013, 25, 2239-2245.

2 J. J. Walish, Y. Kang, R. A. Mickiewicz and E. L. Thomas, Adv. Mater., 2009, 21, 3078-3081.

3 P. M. Resende, R. Sanz, O. Caballero-Calero and M. MartínGonzález, Adv. Opt. Mater., 2018, 6, 1800408.

4 J. A. De La Cruz, Q. Liu, B. Senyuk, A. W. Frazier, K. Peddireddy and I. I. Smalyukh, ACS Photonics, 2018, 5, 2468-2477.

5 Y. Wu, J. Yang, S. Wang, Z. Ling, H. Zhang and B. Wei, Appl. Sci., 2019, 9, 1415.

6 P. Lova, G. Manfredi and D. Comoretto, Adv. Opt. Mater., 2018, 6, 1800730.

7 W. J. Dong, N.-T. Lo, G. H. Jung, J. Ham and J.-L. Lee, Appl. Phys. Lett., 2016, 108, 103902.

8 C. T. Prontera, M. Pugliese, R. Giannuzzi, S. Carallo, M. Esposito, G. Gigli and V. Maiorano, J. Inf. Disp., 2021, 22, 39-47.

9 L. Xu, Y. Yao, N. D. Bronstein, L. Li, A. P. Alivisatos and R. G. Nuzzo, ACS Photonics, 2016, 3, 278-285. 
10 H. I. Elim, B. Cai, Y. Kurata, O. Sugihara, T. Kaino, T. Adschiri, A.-L. Chu and N. Kambe, J. Phys. Chem. B, 2009, 113, 10143-10148.

11 S.-H. Jeong, J.-K. Kim, B.-S. Kim, S.-H. Shim and B.-T. Lee, Vacuum, 2004, 76, 507-515.

12 M. E. Calvo and H. Míguez, Chem. Mater., 2010, 22, 3909-3915.

13 O. Sánchez-Sobrado, M. E. Calvo and H. Míguez, J. Mater. Chem., 2010, 20, 8240-8246.

14 M. E. Calvo, L. González-García, J. Parra-Barranco, A. Barranco, A. Jiménez-Solano, A. R. González-Elipe and H. Míguez, Adv. Opt. Mater., 2015, 3, 171-175.

15 M. Portnoi, T. J. Macdonald, C. Sol, T. S. Robbins, T. Li, J. Schläfer, S. Guldin, I. P. Parkin and I. Papakonstantinou, Nano Energy, 2020, 70, 104507.

16 W. Ma, S. Li, D. Kou, J. L. Lutkenhaus, S. Zhang and B. Tang, Dyes Pigm., 2019, 160, 740-746.

17 P. Lova, Polymers, 2018, 10, 1161.

18 L. Criante and F. Scotognella, J. Phys. Chem. C, 2012, 116, 21572-21576.

19 H. Feng, X. Lu, W. Wang, N.-G. Kang and W. J. Mays, Polymers, 2017, 9, 494.

20 M. Stefik, S. Guldin, S. Vignolini, U. Wiesner and U. Steiner, Chem. Soc. Rev., 2015, 44, 5076-5091.

21 L. Wang and Q. Li, Adv. Funct. Mater., 2016, 26, 10-28.

22 E. P. A. v. Heeswijk, J. J. H. Kloos, J. d. Heer, T. Hoeks, N. Grossiord and A. P. H. J. Schenning, ACS Appl. Mater. Interfaces, 2018, 10, 30008-30013.

23 E. P. A. van Heeswijk, J. J. H. Kloos, N. Grossiord and A. P. H. J. Schenning, J. Mater. Chem. A, 2019, 7, 6113-6119.

24 E. P. A. van Heeswijk, A. J. J. Kragt, N. Grossiord and A. P. H. J. Schenning, Chem. Commun., 2019, 55, 2880-2891.

25 E. P. A. van Heeswijk, T. Meerman, J. de Heer, N. Grossiord and A. P. H. J. Schenning, ACS Appl. Polym. Mater., 2019, 1, 3407-3412.

26 H. S. Lim, J.-H. Lee, J. J. Walish and E. L. Thomas, ACS Nano, 2012, 6, 8933-8939.

27 P. Lova, G. Manfredi, L. Boarino, A. Comite, M. Laus, M. Patrini, F. Marabelli, C. Soci and D. Comoretto, ACS Photonics, 2015, 2, 537-543.

28 L. C. Lohithakshan, V. Geetha and P. Kannan, Opt. Mater., 2020, 110, 110509.

29 C. Mijangos, R. Hernández and J. Martín, Prog. Polym. Sci., 2016, 54-55, 148-182.

30 W. Lee and S.-J. Park, Chem. Rev., 2014, 114, 7487-7556.

31 C. V. Manzano, D. Ramos, L. Pethö, G. Bürki, J. Michler and L. Philippe, J. Phys. Chem. C, 2018, 122, 957-963.

32 A. Santos, J. Mater. Chem. C, 2017, 5, 5581-5599.

33 C. S. Law, S. Y. Lim, A. D. Abell, N. H. Voelcker and A. Santos, Nanomaterials, 2018, 8, 788.

34 J. Martín, M. Martín-González, J. Francisco Fernández and O. Caballero-Calero, Nat. Commun., 2014, 5, 5130.

35 P. M. Resende, E. Gutiérrez-Fernández, M. H. Aguirre, A. Nogales and M. Martín-González, Polymer, 2021, 212, 123145.

36 A. García-Barberá, M. Culebras, S. Roig-Sánchez, C. M. Gómez and A. Cantarero, Synth. Met., 2016, 220, 208-212.
37 A. Ruiz-Clavijo, O. Caballero-Calero and M. MartínGonzález, Nanoscale, 2021, 13, 2227-2265.

38 D. Garlotta, J. Polym. Environ., 2001, 9, 63-84.

39 J. Lunt, Polym. Degrad. Stab., 1998, 59, 145-152.

40 M. Smith, C. Lindackers, K. McCarthy and S. Kar-Narayan, Macromol. Mater. Eng., 2019, 304, 1800607.

41 R. Androsch and M. L. Di Lorenzo, Polymer, 2013, 54, 6882-6885.

42 S. P. Dubey, V. K. Thakur, S. Krishnaswamy, H. A. Abhyankar, V. Marchante and J. L. Brighton, Vacuum, 2017, 146, 655-663.

43 J. P. Kalish, K. Aou, X. Yang and S. L. Hsu, Polymer, 2011, 52, 814-821.

44 P. Klonos, S. Kripotou, A. Kyritsis, G. Z. Papageorgiou, D. Bikiaris, D. Gournis and P. Pissis, Thermochim. Acta, 2015, 617, 44-53.

45 J.-M. Raquez, Y. Habibi, M. Murariu and P. Dubois, Prog. Polym. Sci., 2013, 38, 1504-1542.

46 S. Saeidlou, M. A. Huneault, H. Li and C. B. Park, Prog. Polym. Sci., 2012, 37, 1657-1677.

47 G. Shi, Y. Guan, G. Liu, A. J. Müller and D. Wang, Macromolecules, 2019, 52, 6904-6912.

48 D. Barata, P. Dias, P. Wieringa, C. van Blitterswijk and P. Habibovic, Biofabrication, 2017, 9, 035004.

49 S. A. Munim and Z. A. Raza, J. Porous Mater., 2019, 26, 881-901.

50 M. Ando, H. Kawamura, K. Kageyama and Y. Tajitsu, Jpn. J. Appl. Phys., 2012, 51, 09LD14.

51 S. J. Lee, A. P. Arun and K. J. Kim, Mater. Lett., 2015, 148, 58-62.

52 Q. Jing and S. Kar-Narayan, J. Phys. D: Appl. Phys., 2018, 51, 303001.

53 R. Pan, W. Xuan, J. Chen, S. Dong, H. Jin, X. Wang, H. Li and J. Luo, Nano Energy, 2018, 45, 193-202.

54 M. Smith, Y. Calahorra, Q. Jing and S. Kar-Narayan, APL Mater., 2017, 5, 074105.

55 J. Zhu, L. Jia and R. Huang, J. Mater. Sci.: Mater. Electron., 2017, 28, 12080-12085.

56 G. Mattana, D. Briand, A. Marette, A. Vásquez Quintero and N. F. de Rooij, Org. Electron., 2015, 17, 77-86.

57 M. Atreya, K. Dikshit, G. Marinick, J. Nielson, C. Bruns and G. L. Whiting, ACS Appl. Mater. Interfaces, 2020, 12, 23494-23501.

58 A. E. Ongaro, D. Di Giuseppe, A. Kermanizadeh, A. Miguelez Crespo, A. Mencattini, L. Ghibelli, V. Mancini, K. L. Wlodarczyk, D. P. Hand, E. Martinelli, V. Stone, N. Howarth, V. La Carrubba, V. Pensabene and M. Kersaudy-Kerhoas, Anal. Chem., 2020, 92, 6693-6701.

59 J. Cambiasso, S. Goyanes and S. Ledesma, Opt. Mater., 2015, 47, 72-77.

60 R. Fu, W. Luo, R. Nazempour, D. Tan, H. Ding, K. Zhang, L. Yin, J. Guan and X. Sheng, Adv. Opt. Mater., 2018, 6, 1700941.

61 Y. Cao, K. Nallappan, H. Guerboukha, T. Gervais and M. Skorobogatiy, Opt. Express, 2019, 27, 27663-27681.

62 A. Gierej, M. Vagenende, A. Filipkowski, B. Siwicki, R. Buczynski, H. Thienpont, S. V. Vlierberghe, T. Geernaert, 
P. Dubruel and F. Berghmans, J. Light Technol., 2019, 37, 1916-1923.

63 J. Feng, Q. Jiang, P. Rogin, P. W. de Oliveira and A. del Campo, ACS Appl. Mater. Interfaces, 2020, 12, 20287-20294.

64 M. Fujishima, S. Sakata, M. Kikoku, D. Ogawa and K. Uchida, Chem. Lett., 2007, 36, 1510-1511.

65 R. Xiong, J. Luan, S. Kang, C. Ye, S. Singamaneni and V. V. Tsukruk, Chem. Soc. Rev., 2020, 49, 983-1031.
66 M. Steinhart, R. B. Wehrspohn, U. Gösele and J. H. Wendorff, Angew. Chem., Int. Ed., 2004, 43, 1334-1344.

67 H. Fudouzi and T. Sawada, Langmuir, 2006, 22, 1365-1368. 68 K. Liu, T. A. Schmedake and R. Tsu, Phys. Lett. A, 2008, 372, 4517-4520.

69 P. M. Resende and M. Martín-González, Microporous Mesoporous Mater., 2019, 284, 198-204.

70 B. Abad, J. Maiz and M. Martin-Gonzalez, J. Phys. Chem. C, 2016, 120, 5361-5370. 